\title{
Applicability of the two-particle quantization condition to partially-quenched theories
}

\author{
Zachary T. Draper* and Stephen R. Sharpe ${ }^{\dagger}{ }^{\dagger}$ \\ Physics Department, University of Washington, Seattle, Washington 98195-1560, USA
}

(Received 23 July 2021; accepted 10 August 2021; published 31 August 2021)

\begin{abstract}
Partial quenching allows one to consider correlation functions and amplitudes that do not arise in the corresponding unquenched theory. For example, physical $s$-wave pion scattering can be decomposed into $I=0$ and 2 amplitudes, while, in a partially-quenched extension, the larger symmetry group implies that there are more than two independent scattering amplitudes. It has been proposed that the finite-volume quantization condition of Lüscher holds for the correlation functions associated with each of the twoparticle amplitudes that arise in partially-quenched theories. Using partially-quenched chiral perturbation theory, we show that this proposal fails for those correlation functions for which the corresponding oneloop amplitudes do not satisfy $s$-wave unitarity. For partially-quenched amplitudes that, while being unphysical, do satisfy one-loop s-wave unitarity, we argue that the proposal is plausible. Implications for previous work are discussed.
\end{abstract}

DOI: $10.1103 /$ PhysRevD.104.034510

\section{INTRODUCTION}

Simulations of lattice QCD (LQCD) and related theories naturally separate into the generation of gauge fields and the calculation of quark propagators on these gauge fields. This separation allows one the freedom to "partially quench," which in broadest terms means treating valence and sea quarks differently. This can be done by using different valence- and sea-quark masses or, as we consider here, by keeping valence- and sea-quark masses the same but considering correlation functions-combinations of quark propagators - that cannot arise in QCD. In either case, one obtains extra information about the underlying theory. The important questions are then whether that information is useful, e.g., for extracting physical quantities, and, if so, how the desired output is extracted.

There are many proposals for the extraction of additional information using partially-quenched (PQ) theories, several of which have been applied in practice. Most involve the use of partially-quenched chiral perturbation theory $(\mathrm{PQ} \chi \mathrm{PT})$, which was developed in Ref. [1]. For reviews see Refs. [2,3]. One usage is to aid the chiral extrapolation/ interpolation to the physical point using a range of valence quark masses [4], and this has been widely applied in

\footnotetext{
ztd@uw.edu

†rsharpe@uw.edu
}

Published by the American Physical Society under the terms of the Creative Commons Attribution 4.0 International license. Further distribution of this work must maintain attribution to the author(s) and the published article's title, journal citation, and DOI. Funded by SCOAP ${ }^{3}$. practice. Another example, closely related to the discussion below, is the proposal of Ref. [5] to separately calculate contributions to $\pi^{+} \pi^{+}$correlators with and without quark exchange, so as to determine the lattice-spacing-dependent low-energy coefficients (LECs) of "Wilson $\chi$ PT" (i.e., $\chi$ PT applied to Wilson fermions). One can also use PQ $\chi$ PT to estimate quark-disconnected diagrams from more easily calculated correlators, e.g., in the hadronic vacuum polarization contribution to muonic $g-2$ [6], or in pion scattering [7]. We also note the use of partially quenched theories to study the spectral density of the Dirac operator [8-11] and its relation to random matrix theories [12].

This paper considers the proposals of Refs. [13,14], which concern, respectively, $\pi \pi$ and $K \pi$ scattering in QCD. These works consider PQ extensions of QCD containing additional valence quarks (and corresponding ghost quarks) such that the flavor symmetry group extends from $\mathrm{SU}(2)$ to $\mathrm{SU}(4 \mid 2)$ for $\pi \pi$ scattering, and from $\mathrm{SU}(3)$ to $\mathrm{SU}(4 \mid 1)$ for $K \pi$ scattering. ${ }^{1}$ Within these extensions the scattering amplitudes of pions and kaons (composed of either sea or valence quarks) can be decomposed into irreducible representations (irreps) of the quark flavor symmetry group, which is $\mathrm{SU}(4)$ in both cases. This group is larger than the symmetry group of the unquenched sea quarks, which is $\mathrm{SU}(2)$ and $\mathrm{SU}(3)$, respectively, for the two cases. This implies that the number of irreps, and associated independent amplitudes, is larger in the PQ theory than in

\footnotetext{
${ }^{1}$ The notation here is that the $\mathrm{SU}(n \mid m)$ symmetry corresponds to $n-m$ sea quarks, $m$ valence quarks, and $m$ (commuting) ghost quarks. The flavor symmetry of the unquenched subsector is $\mathrm{SU}(n-m)$.
} 
the unquenched theory. For example, for $s$-wave $\pi \pi$ scattering, there are four independent amplitudes in the quark sector of the PQ theory, corresponding to the symmetric product of two adjoints in SU(4) [15],

$$
(\mathbf{1 5} \times 15)_{\mathrm{sym}}=\mathbf{1}+\mathbf{1 5}+\mathbf{2 0}+\mathbf{8 4},
$$

whereas for unquenched pions in QCD the decomposition contains only two amplitudes, those with isospin 0 and 2 . The physical $I=2$ amplitude is equivalent to the $\mathbf{8 4}$, while the $I=0$ amplitude is equivalent to that obtained from an operator that is a linear combination of the $\mathbf{1}$ with elements of the 15, 20, and $\mathbf{8 4} .^{2}$ The remaining independent amplitudes, which we take to be those in the $\mathbf{1 5}$ and $\mathbf{2 0}$ irreps, are artifacts of the PQ theory, and correspond to linear combinations of Wick contractions that are not present in the unquenched theory.

We focus in this paper on nonsinglet irreps of the quark flavor group, as this simplifies the calculations and yet allows us to make our main points. We make some brief comments on the singlet irrep in the conclusions.

A central claim of Refs. $[13,14]$ is the following: for each of the PQ amplitudes, the corresponding PQ two-particle correlation function determines a spectrum of energies that can be related to the scattering amplitude using the twoparticle quantization condition of Lüscher [16,17]. We stress that this claim consists of two parts: the first that the correlation function can be used to extract a spectrum of states in the usual way, and the second that the resulting spectrum can be analyzed using the two-particle quantization condition. To make these statements concrete, consider the $r=\mathbf{1 5}$ irrep in PQ pion scattering, and let $\mathcal{O}_{r}$ be an operator with these quantum numbers. The first part of the claim is that

$$
\left\langle\mathcal{O}_{r}(\tau) \mathcal{O}_{r}^{\dagger}(0)\right\rangle=\sum_{n=0}^{\infty} c_{n} e^{-E_{n} \tau},
$$

where the expectation value is in the underlying PQ theory, $\tau$ is Euclidean time, the coefficients $c_{n}$ are real and positive, and the energies $E_{n}$ are real and bounded below. For convenience we order them $E_{0} \leq E_{1} \leq E_{2}$, etc. The second part of the claim is that we can insert the resulting energies $E_{n}$ into Lüscher's quantization condition and correctly obtain the scattering amplitude in the corresponding irrep. We know that this procedure is valid for the $\mathbf{8 4}$ irrep, as it corresponds to a physical correlation function in QCD. The issue is whether it remains valid for the irreps available only in the PQ theory.

\footnotetext{
${ }^{2}$ The linear combination is $\frac{1}{5} \mathbf{1}+\frac{1}{2} \mathbf{1 5}+\frac{1}{4} \mathbf{2 0}+\frac{1}{20} \mathbf{8 4}$, as can be seen by considering the quark contractions. That this leads to a physical amplitude is not immediately clear from the group theory, but follows from the fact that operators living entirely in the unquenched sector are free from PQ artifacts.
}

If correct, this proposal is quite powerful, as, combined with $\mathrm{PQ} \chi \mathrm{PT}$, it allows the determination of results from types of contractions that are difficult to calculate numerically from those that are simpler to evaluate $[7,13,14,18]$. It is, however, prima facie surprising, since both the existence of a normal spectral decomposition and the derivation of the two-particle quantization condition rely on unitarity, which the PQ theory violates $[1,19]$.

In this work we introduce a necessary (but not sufficient) criterion for whether this proposal holds in a given PQ irrep. We apply it in detail to $\pi \pi$ scattering, which allows us to develop alternative criteria for when the proposal will fail. We then apply these criteria to other systems.

The remainder of this paper is organized as follows. Section II describes the setup within $\mathrm{PQ} \chi \mathrm{PT}$ that we use to formulate our initial criterion. Section III presents the results of the application of this criterion to $\pi \pi$ scattering. Section IV interprets these results using quark-line diagrams, presents two alternative versions of the criterion, and discusses the extent to which our necessary criterion is actually sufficient. Section V discusses a few applications of our criteria and describes their implications for previous work. Finally, Sec. VI presents some closing comments.

\section{THEORETICAL SETUP}

We follow closely the methodology of Ref. [5], although here we work in the continuum. We introduce two valence quarks in addition to the physical up and down quark, as well as two ghost quarks, with all quarks and ghost quarks having a common mass $m$. Thus the graded flavor symmetry group is $\mathrm{SU}(4 \mid 2) .{ }^{3}$ This is the same theoretical setup as used in Refs. $[7,13]$. We label the quark fields $q_{i}$, $i=1-4$, with $q_{1}=u$ and $q_{2}=d$ being thought of as the sea quarks, although this choice is purely conventional given the exact $\mathrm{SU}(4)$ quark flavor symmetry. The two ghost quarks are labeled $\tilde{q}_{5}$ and $\tilde{q}_{6}$. Using this collection of fields we can write down the PQQCD functional integral in Euclidean space as described in Ref. [1].

The long-distance properties of this theory are described by PQ $\chi$ PT. The degrees of freedom of this effective theory are the pseudo-Goldstone (PG) boson and fermion fields, which are collected into the following straceless ${ }^{4} 6 \times 6$ matrix $[1,20]$,

\footnotetext{
${ }^{3}$ Superficially, this differs from the setup in Ref. [5], where four valence quarks and corresponding ghost quarks were introduced, leading to an $\mathrm{SU}(6 \mid 4)$ flavor symmetry. For the purposes of our calculations, however, the difference is trivial: both calculations build the required operators out of four degenerate quark fields, leading to identical quark-level contractions and $\chi \mathrm{PT}$ results. The additional valence quarks were introduced in Ref. [5] in order to calculate a different quantity, as described in Appendix B of that work.

${ }^{4}$ We use "straceless" as a shorthand for having a vanishing supertrace, and we refer to the latter as "strace" or "str."
} 


$$
\Pi=\frac{1}{\sqrt{2}}\left(\begin{array}{cccccc}
\frac{1}{\sqrt{2}} \pi_{0}+\frac{1}{2} \eta_{4}+\frac{1}{2} \phi_{1} & \pi_{12} & \pi_{13} & \pi_{14} & \omega_{15} & \omega_{16} \\
\pi_{21} & -\frac{1}{\sqrt{2}} \pi_{0}+\frac{1}{2} \eta_{4}+\frac{1}{2} \phi_{1} & \pi_{23} & \pi_{24} & \omega_{25} & \omega_{26} \\
\pi_{31} & \pi_{32} & \frac{1}{\sqrt{2}} \pi_{0}^{\prime}-\frac{1}{2} \eta_{4}+\frac{1}{2} \phi_{1} & \pi_{34} & \omega_{35} & \omega_{36} \\
\pi_{41} & \pi_{42} & \pi_{43} & -\frac{1}{\sqrt{2}} \pi_{0}^{\prime}-\frac{1}{2} \eta_{4}+\frac{1}{2} \phi_{1} & \omega_{45} & \omega_{46} \\
\omega_{51} & \omega_{52} & \omega_{53} & \omega_{54} & \frac{1}{\sqrt{2}} \phi_{0}+\phi_{1} & \phi_{56} \\
\omega_{61} & \omega_{62} & \omega_{63} & \omega_{64} & \phi_{65} & -\frac{1}{\sqrt{2}} \phi_{0}+\phi_{1}
\end{array}\right) .
$$

Here $\pi_{0}$ is the usual neutral pion field (in isosymmetric QCD), with $\pi_{0}^{\prime}$ being the corresponding valence field, while $\eta_{4}$ is the four flavor generalization of the $\eta$ meson in QCD. The 15 fields in the quark sector of $\Pi$ fill out the SU(4) adjoint irrep of PG bosons (PGBs). The $\omega_{i j}$ are PG fermion (PGF) fields corresponding to quark-ghost combinations, while the $\phi_{0}, \phi_{i j}$, and $\phi_{1}$ are PG ghost bosons (with propagators having an unphysical overall sign).

We will only need the leading order (LO) chiral Lagrangian for this theory, which is given in Euclidean space by

$$
\mathcal{L}_{\mathrm{LO}}=\frac{f^{2}}{4} \operatorname{str}\left(\partial_{\mu} \Sigma^{\dagger} \partial_{\mu} \Sigma\right)-\frac{m B_{0} f^{2}}{2} \operatorname{str}\left(\Sigma^{\dagger}+\Sigma\right),
$$

where $f$ and $B_{0}$ are the usual LO LECs, with the former defined in the small $f$ convention, and

$$
\Sigma=\exp (2 i \Pi / f) .
$$

The masses of all the PGBs and PGFs are given, at LO, by

$$
M_{\pi}^{2}=2 B_{0} m
$$

We note also that, because all the quarks and ghost quarks are degenerate, the PG propagators all contain only single poles, with no double-pole contributions $[4,20]$.

The relation of the pion fields in $\chi \mathrm{PT}$ to operators in the underlying theory can be worked out using the spurion method. The resulting correspondence is given for $j, k=$ 1-4 by (see e.g., Ref. [5]) $\bar{q}_{j} \gamma_{5} q_{k}(x)=c\left(\Sigma-\Sigma^{\dagger}\right)_{k j}$, with $c$ a known constant that will cancel in the ratios considered below. Choosing $j \neq k$ for simplicity, the right-hand side of this relation is proportional to $\pi_{k j}$, up to chiral corrections proportional to $\pi^{2} / f^{2}$. As discussed in Sec. C.1 of Ref. [5], these correction terms lead to subleading contributions to the correlation functions that lie beyond the accuracy that we consider. Thus we effectively have the correspondence $\pi_{k j} \sim \bar{q}_{j} \gamma_{5} q_{k}$, and, similarly, $\pi^{0} \sim \frac{1}{\sqrt{2}}\left(\bar{q}_{1} \gamma_{5} q_{1}-\bar{q}_{2} \gamma_{5} q_{2}\right)$ and $\eta_{4} \sim \frac{1}{2}\left(\bar{q}_{1} \gamma_{5} q_{1}+\bar{q}_{2} \gamma_{5} q_{2}-\bar{q}_{3} \gamma_{5} q_{3}-\bar{q}_{4} \gamma_{5} q_{4}\right)$, with a common constant of proportionality. Using this correspondence, the definitions given below apply both in the underlying theory, PQQCD, and in the effective theory, $\mathrm{PQ} \chi \mathrm{PT}$, and we will move between these two representations as needed.

The quantities that we calculate are finite-volume correlation functions involving operators coupling to pairs of PGB fields. These are built from fields having zero spatial momentum, e.g.,

$$
\tilde{\pi}_{12}(\tau)=\int_{L} d^{3} x \pi_{12}(x, \tau)
$$

where the subscript $L$ indicates that the integral is over a cubic box of side $L$. We assume periodic boundary conditions (PBC) in spatial directions on the PGBs and PGFs, which follow if the quark fields satisfy PBC, as it is standard in LQCD simulations. We take the Euclidean time extent to be infinite. We then construct two-PGB operators by forming linear combinations of the building blocks

$$
\tilde{\pi}_{i j}(\tau) \tilde{\pi}_{k \ell}(\tau),
$$

chosen to have definite flavor quantum numbers. Explicit examples of such operators are given in the following section.

In a physical, unitary theory, we can describe the properties of these operators as follows. In the absence of interactions, such operators would simply couple to two pions at rest. In the presence of interactions, however, they couple to all states with vanishing total three-momentum, $\boldsymbol{P}=0$, that have the same flavor as the operator. In particular, these operators couple to the lightest two-PGB state of the chosen flavor. We refer to this as the threshold state, with energy $E_{0}$. Of particular interest is the energy shift $\delta E_{0}=E_{0}-2 M_{\pi}$, for this is given by the threshold expansion of the Lüscher quantization condition [16]

$$
\delta E_{0}=-\frac{\mathcal{A}_{\mathrm{th}}}{4 M_{\pi}^{2} L^{3}}\left[1+c_{1} \frac{\mathcal{A}_{\mathrm{th}}}{16 \pi M_{\pi} L}+\mathcal{O}\left(L^{-2}\right)\right] .
$$


Here $\mathcal{A}_{\text {th }}$ is the scattering amplitude at threshold in the flavor channel under consideration, which is related to the scattering length $a_{0}$ by $^{5}$

$$
\mathcal{A}_{\mathrm{th}}=16 \pi M_{\pi} a_{0},
$$

while $c_{1} \approx-2.84$ is a known geometric constant. We stress that both $\mathcal{A}_{\text {th }}$ and $a_{0}$ are infinite-volume quantities. Higherorder terms in $1 / L$ in Eq. (9) are known, but will not be needed here. There are also (in general unknown) corrections to Eq. (9) that are exponentially suppressed in $M_{\pi} L$. In our theoretical calculation these can always be made arbitrarily small, compared to the power-law dependence that we control, by taking $L$ large enough, and henceforth we ignore them.

To obtain $\delta E_{0}$ from finite-volume correlators, it is convenient to use ratios of the two-PGB correlator to the square of single-particle correlators. Let $\mathcal{O}_{r}(\tau)$ denote (as in the Introduction) a two-PGB operator transforming in the flavor irrep $r$. Then we consider

$$
R_{r}(\tau)=\frac{\left\langle\mathcal{O}_{r}(\tau) \mathcal{O}_{r}^{\dagger}(0)\right\rangle}{\left\langle\tilde{\pi}_{12}(\tau) \tilde{\pi}_{21}(0)\right\rangle^{2}},
$$

where the choice of flavor of the single-PGB correlator in the denominator is arbitrary, because all such correlators fall asymptotically as $\exp \left(-M_{\pi}|\tau|\right)$, due to the $\mathrm{SU}(4)$ flavor symmetry. For a physical theory, the ratio behaves at large $|\tau|$ as

$R_{r}(\tau)=Z_{r} e^{-\delta E_{0, r}|\tau|}+$ excited-state contributions,

where $\delta E_{0, r}$ is the threshold energy shift in the given flavor irrep and $Z_{r}$ is a positive real constant. In an analytic calculation, the contribution from excited states can be separated by hand, since they have a distinctive exponential falloff, with $\delta E_{i} \propto 1 / L^{2}$ asymptotically. The first part of our criterion is then to check that what remains after excited-state contributions have been dropped is an exponential. More precisely, we test that the ratio contains the first three terms in the expansion,

$$
R_{r}(\tau)=Z_{r}\left[1-|\tau| \delta E_{0, r}+\frac{\tau^{2}}{2}\left(\delta E_{0, r}\right)^{2}\right]+\cdots,
$$

where the ellipsis represents higher-order terms in $\tau$ as well as the excited-state contributions.

The second part of our criterion tests whether the threshold expansion, Eq. (9), holds to first nontrivial order in $1 / L$. It turns out that one can test this, as well as Eq. (13), by carrying out a next-to-leading-order (NLO) calculation of the correlator ratio in $\chi \mathrm{PT}$. To see how this works, we

\footnotetext{
${ }^{5}$ Here we are using the normalization appropriate for distinguishable particles.
}

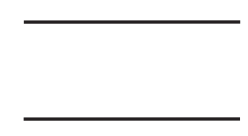

(a)

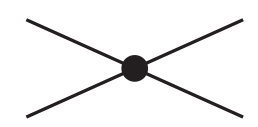

(b)

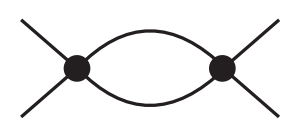

(c)
FIG. 1. PQ $\chi \mathrm{PT}$ diagrams contributing to $R_{r}(\tau)$, Eq. (11). Time runs horizontally. Solid lines are propagators of PGBs and PGFs, and filled circles represent vertices obtained from the expansion of $\mathcal{L}_{\mathrm{LO}}$, Eq. (4). These are the diagrams needed to implement the test discussed in the text.

need several results that follow from the similar calculation carried out in Ref. [5]. The relevant diagrams are those shown in Fig. 1. Figure 1(a) gives the leading, $\tau$-independent term in the exponential in Eq. (13), and one can choose the normalization of $\mathcal{O}_{r}$ such that $Z_{r}=1$ at this stage. Figure 1(b) leads to a term linear in $|\tau|$, in which $\delta E_{0, r}=-\mathcal{A}_{\mathrm{th}, r}^{\mathrm{LO}} /\left(4 M_{\pi}^{2} L^{3}\right)$, which is the form expected at this order from combining Eqs. (9) and (13). This result will hold for all irreps $r$, since the diagrams contributing to the finite-volume correlator and the scattering amplitude are essentially the same. Figure 1(b) also leads to corrections to $Z_{r}$ that are proportional to $1 /\left(f^{2} M_{\pi} L^{3}\right)$.

The key diagram for our test is that of Fig. 1(c). In particular, for a physical theory, it leads both to the $\tau^{2}$ term in Eq. (13) with the LO form for the energy shift, $\delta E_{0, r}=-\mathcal{A}_{\mathrm{th}, r}^{\mathrm{LO}} /\left(4 M_{\pi}^{2} L^{3}\right)$, and to the first $1 / L^{4}$ correction linear in $|\tau|$, i.e.,

$$
\delta E_{0, r} \supset-c_{1} \frac{\left(\mathcal{A}_{\mathrm{th}, r}^{\mathrm{LO}}\right)^{2}}{64 \pi M_{\pi}^{3} L^{4}} .
$$

It is these contributions that will turn out to have the wrong form for some irreps in the PQ theory.

Figure 1(c) also contributes to the leading $1 / L^{3}$ part of the coefficient of $|\tau|$. Together with diagrams containing NLO vertices, as well as self-energy diagrams, this leads to the expected form of the linear term at this order [5]:

$$
R_{r}(\tau) \supset|\tau| \frac{\mathcal{A}_{\mathrm{th}, r}^{\mathrm{NLO}}}{4 M_{\pi}^{2} L^{3}},
$$

where $\mathcal{A}_{\mathrm{th}, r}^{\mathrm{NLO}}$ is the complete threshold amplitude through NLO. As at LO, this result holds for all irreps $r$, because the diagrams that contribute to the $|\tau| / L^{3}$ term are the same as those leading to infinite-volume scattering. The only difference is that the former involve momentum sums, while the latter contain integrals, but the difference can be shown to be subleading, behaving as $|\tau| / L^{4}$, and leads to the $c_{1}$ term in Eq. (14) [5]. As stressed in Ref. [5], the result that the coefficient of $|\tau| / L^{3}$ gives the scattering amplitude is a finite volume version of the Lehman-SymanzikZimmermann reduction theorem. 
Pulling all this together, we now state our criterion for whether a particular PQ channel can be treated as if it were physical.

C1: In order for a channel to be described by the twoparticle quantization condition, one must find the following result ${ }^{6}$ if one calculates the diagrams of Fig. 1:

$$
R_{r}(\tau)=1+|\tilde{\tau}|\left[\mathcal{A}_{\mathrm{th}, r}^{\mathrm{LO}}+c_{1} \frac{\left(\mathcal{A}_{\mathrm{th}, r}^{\mathrm{LO}}\right)^{2}}{16 \pi M_{\pi} L}\right]+\frac{\tilde{\tau}^{2}}{2}\left(\mathcal{A}_{\mathrm{th}, r}^{\mathrm{LO}}\right)^{2}+\cdots,
$$

where

$$
\tilde{\tau}=\frac{\tau}{4 M_{\pi}^{2} L^{3}},
$$

and the ellipsis indicates terms of higher order in $|\tilde{\tau}|$ and $1 / L, N L O$ contributions to $\mathcal{A}_{\mathrm{th}, r}$ in the $|\tilde{\tau}|$ term, as well as excited state contributions.

In other words, we can focus on the terms that lead to factors of $\mathcal{A}_{\mathrm{th}, r}^{\mathrm{LO}}$, with a full NLO calculation of this amplitude not required. Evaluating Fig. 1(b) gives the result for $\mathcal{A}_{\mathrm{th}, r}^{\mathrm{LO}}$, while Fig. 1(c) gives the $c_{1}$ and $\tau^{2}$ terms, which must have the dependence on $\mathcal{A}_{\mathrm{th}, r}^{\mathrm{LO}}$ given in Eq. (16). We also note that one can check the calculation of Fig. 1(b) by evaluating the amplitude $\mathcal{A}_{\mathrm{th}, r}^{\mathrm{LO}}$ directly in infinite volume.

Passing this test is necessary in order that a channel can be treated as physical, but it is clearly not sufficient, since higher order terms in $\tau$ and in the threshold expansion of Eq. (9) are not checked. In addition, the test considers only the threshold state, while a complete test would also consider excited states. Possible generalizations are discussed below in Sec. IV.

\section{PQ $\chi$ PT CALCULATION OF $\pi \pi$ CORRELATORS}

We perform the test described in the previous section for the $\mathbf{2 0}$ and $\mathbf{1 5}$ irreps, which are the two that are absent in the unquenched theory. For comparison we also include the $\mathbf{8 4}$ irrep. Examples of two-pion operators transforming in these irreps are

$$
\begin{aligned}
& \mathcal{O}_{\mathbf{8 4}}=\frac{1}{\sqrt{2}}\left(\tilde{\pi}_{12} \tilde{\pi}_{34}+\tilde{\pi}_{14} \tilde{\pi}_{32}\right), \\
& \mathcal{O}_{\mathbf{2 0}}=\frac{1}{\sqrt{2}}\left(\tilde{\pi}_{12} \tilde{\pi}_{34}-\tilde{\pi}_{14} \tilde{\pi}_{32}\right),
\end{aligned}
$$

\footnotetext{
${ }^{6}$ Here we are assuming the above-described normalization choice for $\mathcal{O}_{r}$.
}

$$
\mathcal{O}_{\mathbf{1 5}}=\frac{1}{\sqrt{3}}\left(\tilde{\pi}_{13} \tilde{\pi}_{32}+\tilde{\pi}_{14} \tilde{\pi}_{42}+\tilde{\pi}_{12} \tilde{\eta}_{4}\right)
$$

The normalization factors are chosen as described in the previous section, i.e., such that the leading term in $R_{\tau}$ is unity. In the notation of Ref. [13] these three irreps are labeled $\alpha, \beta$, and $\gamma$, respectively. The LO threshold amplitudes for these irreps can be read off from the results for scattering lengths given in Ref. [13] (noting that the scattering length in that work is defined with opposite sign to ours), yielding

$$
\mathcal{A}_{\mathrm{th}, \mathbf{8 4}}^{\mathrm{LO}}=-\frac{M_{\pi}^{2}}{f^{2}}, \quad \mathcal{A}_{\mathrm{th}, \mathbf{2 0}}^{\mathrm{LO}}=\frac{M_{\pi}^{2}}{f^{2}}, \quad \mathcal{A}_{\mathrm{th}, \mathbf{1 5}}^{\mathrm{LO}}=\frac{7}{2} \frac{M_{\pi}^{2}}{f^{2}} .
$$

The implementation of the criterion $\mathrm{C} 1$ for the $\mathbf{8 4}$ and $\mathbf{2 0}$ irreps can be done by combining results from Ref. [5]. In the notation of that work

$$
R_{\mathbf{8 4} / \mathbf{2 0}}(\tau)=R_{\mathcal{D}}(\tau) \pm R_{\mathcal{S}}(\tau),
$$

where $R_{\mathcal{D}}$ involves the double-loop (or "direct" or $D$ for short) Wick contraction in the numerator of the ratio, while $R_{\mathcal{S}}$ involves the single loop (" $S$ ") contraction, which are shown in Figs. 2(a) and 2(b), respectively. In Refs. [7,13], the latter contraction is labeled $C$ for crossed. The results for $D(\tau)$ and $S(\tau)$ at NLO in PQ $\chi \mathrm{PT}$ are given in Eqs. (104) and (105) of Ref. [5], including the effect of discretization errors for Wilson-like fermions. Setting all LECs associated with discretization errors to zero, and choosing $\tau>0$ as in Ref. [5], the results read

$$
\begin{gathered}
R_{\mathcal{D}}(\tau)=1+\tilde{\tau} \mathcal{D}+\left(\frac{M_{\pi}^{2}}{f^{2}}\right)^{2}\left[\tilde{\tau} \frac{c_{1}}{16 \pi M_{\pi} L}+\frac{\tilde{\tau}^{2}}{2}\right]+\cdots, \\
R_{\mathcal{S}}(\tau)=\tilde{\tau} \mathcal{S}+\cdots .
\end{gathered}
$$

Here $\mathcal{D}$ and $\mathcal{S}$ are the NLO PQ threshold amplitudes associated with the $D$ and $S$ contractions, and are given in Eqs. (106) and (107), respectively, of Ref. [5]. We only need their LO contributions, which are

$$
\mathcal{D}^{\mathrm{LO}}=0, \quad \mathcal{S}^{\mathrm{LO}}=-\frac{M_{\pi}^{2}}{f^{2}} .
$$

Finally, we form the combinations given in Eq. (22), obtaining (again for $\tau>0$ )

$R_{\mathbf{8 4} / \mathbf{2 0}}(\tau)=1+\tilde{\tau}(\mathcal{D} \pm \mathcal{S})+\left(\frac{M_{\pi}^{2}}{f^{2}}\right)^{2}\left[\tilde{\tau} \frac{c_{1}}{16 \pi M_{\pi} L}+\frac{\tilde{\tau}^{2}}{2}\right]+\cdots$.

Since $(\mathcal{D} \pm \mathcal{S})^{\mathrm{LO}}=\mp M_{\pi}^{2} / f^{2}$, we see that the results for both irreps satisfy the criterion C1, i.e., Eq. (16) with the 


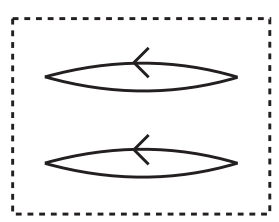

(a) $D$

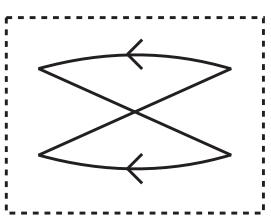

(b) $S$

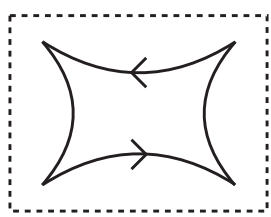

(c) $R$

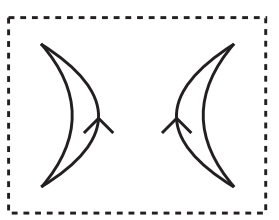

(d) $V$

FIG. 2. Types of Wick contraction that contribute to the two-pion correlators, $R_{r}(\tau)$. Time runs horizontally. Solid lines represent quark propagators, while the dashed box is a reminder that the correlators are evaluated in finite spatial volumes.

LO amplitudes given in Eq. (21). ${ }^{7}$ This is as expected for the physical $r=\mathbf{8 4}$ channel, while, for the $r=\mathbf{2 0}$ channel, it supports the proposal of Ref. [13] that one can apply the quantization condition to this unphysical channel.

Now we turn to the $\mathbf{1 5}$ irrep, for which a new calculation is needed. In terms of valence quark Wick contractions, one needs not only $D$ and $S$ contractions but also the "rectangle" or $R$ contraction shown in Fig. 2(c). We find, using $\mathcal{O}_{15}$ from Eq. (20), that

$$
R_{15}(\tau)=R_{\mathcal{D}}(\tau)-\frac{1}{2} R_{\mathcal{S}}(\tau)+3 R_{\mathcal{R}}(\tau)
$$

in agreement with Ref. [13]. Evaluating the diagrams in Fig. 1, we obtain (with $\tilde{\tau}>0$ )

$$
\begin{aligned}
R_{15}(\tau)= & 1+\tilde{\tau} \frac{7 M_{\pi}^{2}}{2 f^{2}}+\underbrace{\left[\frac{49}{4}-6-\frac{3}{4}\right]}_{11 / 2}\left(\frac{M_{\pi}^{2}}{f^{2}}\right)^{2} \\
& \times\left[\tilde{\tau} \frac{c_{1}}{16 \pi M_{\pi} L}+\frac{\tilde{\tau}^{2}}{2}\right]+\cdots,
\end{aligned}
$$

where the ellipsis has the same meaning as in Eq. (16). The calculation leading to Eq. (28) is straightforward, as the $c_{1} \tilde{\tau}$ and $\tilde{\tau}^{2}$ terms essentially pick out contributions that are part of the square of the tree-level threshold amplitude. The only subtlety is keeping track of which two-particle intermediate states contribute, and of cancellations between contributions involving PG bosons and fermions. We have broken down the contribution to the final term in Eq. (28) into that from the following intermediate states: $\pi_{13} \pi_{32}, \pi_{14} \pi_{42}$, and $\pi_{12} \eta_{4}$ [leading to the unquenched result $49 / 4=(7 / 2)^{2}$ ], $\omega_{15} \omega_{52}$ and $\omega_{16} \omega_{62}$ (leading to the -6 ), and $\pi_{12} \phi_{1}$ (leading to the $-3 / 4)$. We have obtained these results using both the straceless form of $\Pi$ given in Eq. (3) and an approach in which one projects out the stracefull part by introducing a $\Phi_{0}^{2}$ term [20].

Equation (28) is the main new result of this paper. It is manifestly inconsistent with the form of Eq. (16), because the coefficient of the $\tilde{\tau}^{2}$ and $c_{1} \tilde{\tau}$ contributions is not the

\footnotetext{
${ }^{7}$ This result in fact holds even if we keep all contributions from the LO LECS associated with discretization errors.
}

square of the LO threshold amplitude (which itself is correctly determined from the coefficient of $\tilde{\tau}$ ). Thus, the $r=\mathbf{1 5}$ channel fails our test: $R_{\mathbf{1 5}}(\tau)$ cannot be given by a single exponential at long times, and the two-particle quantization condition cannot be used.

\section{DIAGRAMMATIC INTERPRETATION AND ALTERNATIVE CRITERIA}

To gain more understanding of why the $\mathbf{1 5}$ irrep does not satisfy our criterion, while the $\mathbf{2 0}$ does, we consider the quark-line diagrams ${ }^{8}$ associated with the corresponding contributions to Fig. 1(c). These are shown for the 15 irrep in Fig. 3. The key observation is that, for $r=\mathbf{2 0}$, only diagrams of the type of Fig. 3(a) appear, with the four lines having different flavors. This difference arises simply because there can be no "annihilation" of quark flavors for $r=20$, which is why the $R$-type Wick contraction, Fig. 2(c), is not present. This absence implies that there is no violation of unitarity in the $s$-channel in the one-loop diagrams, and it is this form of unitarity (which we refer to as $s$-channel unitarity) that the exponential time dependence and the derivation of the quantization condition depend on.

Conversely, for $r=15$, the appearance of $s$-channel loops involving either ghost quarks [Fig. 3(d)] or neutral PGBs with negative norms such as $\phi_{0}$ [Figs. 3(e) and 3(f)] suggests that $s$-channel unitarity is violated. We emphasize, however, that it is not sufficient to look at the diagrams alone, as the $s$-channel-unitarity-violating contributions can cancel. This indeed happens in the $I=0$ channel, for which there are both $R$ and $V$ Wick contractions [Figs. 2(c) and 2(d)], and thus PQ diagrams involving ghost quarks and negative norm PGBs. Nevertheless, we know that these unphysical contributions must cancel, as this channel is physical.

Putting this together, the following diagrammatic version of the criterion seems highly plausible:

C2: A channel that is not equivalent to a physical correlator will not be described by the two-particle quantization condition if intermediate ghost quarks

\footnotetext{
${ }^{8}$ For more discussion of quark-line diagrams see Ref. [2].
} 


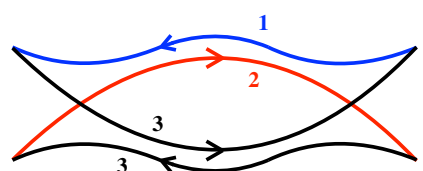

(a)

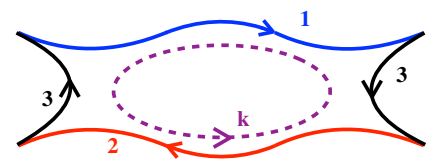

(d)

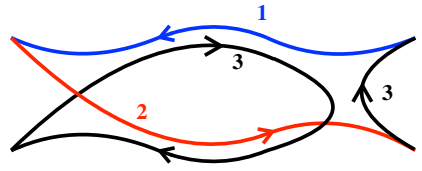

(b)

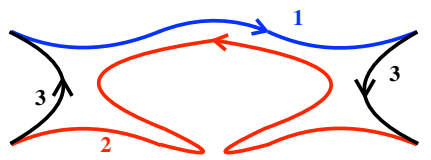

(e)

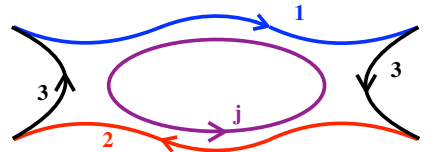

(c)

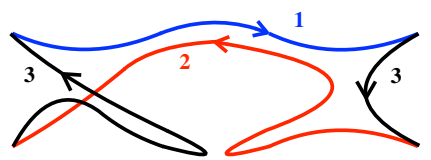

(f)

FIG. 3. Classes of quark-line diagrams contributing to Fig. 1(c) for the $\mathbf{1 5}$ irrep. The diagrams trace the flavor indices of the quarks and antiquarks (or their ghost partners) in the PQ $\chi \mathrm{PT}$ diagrams. Flavors are indicated by colors and numerical labels, with $j=1-4$ in (c) and $k=5-6$ in (d). Dashed lines indicate ghost-quark flavors. In diagrams (e) and (f) the hairpin vertices implicitly contain an infinite sum of intermediate loops of sea quarks (with the valence and ghost loops canceling).

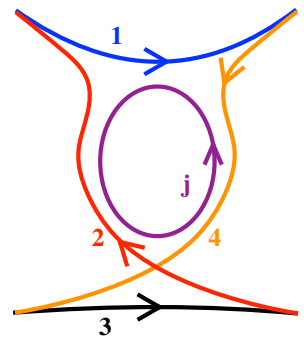

(a)

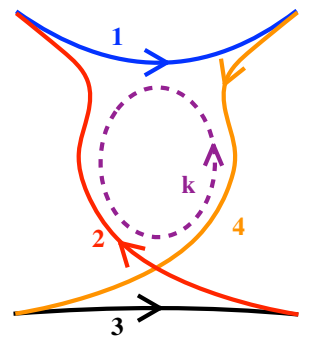

(b)

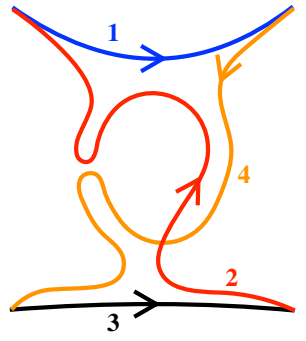

(c)

FIG. 4. Examples of quark-line diagrams contributing to the $t$-channel loops for scattering in the 20 irrep. Notation as in Fig. 3.

appear in (the quark-line diagrams of) s-channel twoparticle loops.

This criterion correctly selects the $\mathbf{1 5}$ irrep as the single PQ nonsinglet $\pi \pi$ channel that is problematic. We have not included negative norm PGBs in the statement of $\mathrm{C} 2$ as it requires explicit calculations to determine whether they are present, and we are aiming here for a simplified criterion. We suspect, however, that nothing is lost by this omission, since, in our experience, diagrams with intermediate ghost quarks and negative norm PGBs come in tandem. C2 is potentially stronger than our original criterion, $\mathrm{C} 1$, as it applies away from threshold. We have, however, not found a general proof of $\mathrm{C} 2$, since this requires knowing that the total contribution from certain classes of quark-line diagrams does not cancel, and this can, in general, only be determined by an explicit calculation, leading us back to $\mathrm{C} 1$.

These diagrammatic considerations lead to another version of the criterion. To explain this, we need to distinguish between unitarity in the $s, t$, and $u$ channels. As noted above, we refer to the standard form of unitarity for an amplitude $\mathcal{T}(s, t, u)$ in the physical domain $\left(s \geq 4 M_{\pi}^{2}\right.$ and $\left.t, u \leq 0\right)$ as $s$-channel unitarity. One can also apply unitarity in the $t$ channel by continuing the amplitude to the appropriate kinematic domain, $t \geq 4 M_{\pi}^{2}$ and $u, s \leq 0$, and similarly for the $u$ channel. An important point to keep in mind is that PQ amplitudes can satisfy unitarity in some channels but not in others. For example, the amplitude $\mathcal{A}_{20}$ satisfies $s$-channel unitarity, but violates $t$ - and $u$-channel unitarity. This is illustrated in Fig. 4, where examples of quark line diagrams contributing to $t$-channel loops are shown. The presence of Figs. 4(b) and 4(c) indicates the violation of $t$-channel unitarity.

Using this terminology, the third version of the criterion is

C3: A channel is not described by the two-particle quantization condition if the (infinite-volume) amplitude $\mathcal{A}_{r}$ violates s-channel unitarity. ${ }^{9}$

\footnotetext{
${ }^{9}$ We stress that we are only considering energies below the first inelastic threshold, e.g., below the four pion threshold for $\pi \pi$ scattering. Above this, the two-particle quantization condition itself breaks down and the question becomes moot.
} 
Again, this correctly picks out the $\mathbf{1 5} \pi \pi$ irrep. The simplest argument for $\mathrm{C} 3$ is that $s$-channel unitarity plays a crucial role in the derivation of the two-particle quantization condition, as is particularly clear from the derivation of Ref. [21]. C3 is stronger than the original form, C1, as the latter involves unitarity applied only infinitesimally above threshold, while C3 applies for all energies up to the first inelastic threshold.

To study C3 in $\chi$ PT requires at least a NLO calculation, since loops are required for the unitarity condition to have nontrivial content. Indeed, from the NLO results for PQ $\pi \pi$ amplitudes in Ref. [7], one can see that $s$-channel unitarity is violated by $\mathcal{A}_{15}^{\mathrm{NLO}}$. In general, $\mathrm{C} 3$ is harder to implement than $\mathrm{C} 1$, as it requires a more extensive calculation in $\chi \mathrm{PT}$.

We end this section by discussing whether the criteria that we have introduced are actually sufficient rather than necessary. We suspect that this is the case, although to demonstrate this would require an all-orders analysis in $\chi \mathrm{PT}$, which is beyond the scope of this work. The point is that higher order terms in the exponential in $\tau$, and in the threshold expansion of the quantization condition, are built up by a sequence of intermediate two-particle $s$-channel loops, as is clear, respectively, from the calculations of Ref. [5] and the derivation of Ref. [21]. The corresponding quark-line diagrams for any irrep that passes the criteria will only involve generalizations of Fig. 3(a), in which quarks or antiquarks are repeatedly exchanged. In particular, there can be no annihilation diagrams at any order in $\chi \mathrm{PT}$. The unphysical contributions in $t$ and $u$ channels, such as those in Fig. 4, will be present, but will appear in all vertices, allowing exponentiation.

\section{APPLICATIONS AND IMPLICATIONS FOR PREVIOUS WORK}

We first summarize our results for the $\pi \pi$ system. In the PQ theory there are three flavor nonsinglet channels, of which one (the 84) is equivalent to a physical channel (with $I=2$ ), which thus automatically satisfies our criteria. Of the unphysical channels, one satisfies our criteria (20), while the other does not (15).

What are the implications for the application presented in Ref. [13]? In that work, the quantization condition was first applied to LQCD results in the $\mathbf{8 4}$ and $\mathbf{2 0}$ channels, and this application is thus not affected by the problem noted here. The results obtained were then used to predict the finitevolume energy spectrum in the $\mathbf{1 5}$ and $I=0$ channels, while our analysis shows that there is no well-defined spectrum in the $\mathbf{1 5}$ channel.

We next turn to the $K \pi$ system, which has been analyzed in this context in Refs. $[14,18]$. Since there are now three light quarks, the isospin symmetry generalizes from $\mathrm{SU}(2)$ in the physical theory to $\mathrm{SU}(3)$ in the PQ version, which is the light quark subgroup of the graded $\mathrm{SU}(4 \mid 1)$ symmetry.
This $\mathrm{SU}(3)$ is exact (assuming $m_{u}=m_{d}$ ), and should not be confused with the approximate $\mathrm{SU}(3)$ in QCD involving the strange quark. There are two physical channels, with $I=3 / 2$ and $1 / 2$, while there are three PQ channels, corresponding to the $\mathrm{SU}(3)$ decomposition $\mathbf{8} \times \mathbf{3}=$ $\mathbf{1 5}+\overline{\mathbf{6}}+\mathbf{3}$. These three channels are labeled $\alpha, \beta$, and $\gamma$, respectively, in Ref. [14]. Of these, only the $\overline{\mathbf{6}}$ is unphysical, with the $\mathbf{1 5}$ being equivalent to the physical $I=3 / 2$ channel, and the 3 to that with $I=1 / 2$.

Applying the criterion $\mathrm{C} 2$ to the $\overline{\mathbf{6}}$ channel, we find that it passes our test, because it involves Wick contractions only of types $D$ and $S$, and is thus completely analogous to the 20 irrep for $\pi \pi$ scattering. Therefore, the numerical application presented in Ref. [18], which uses the $\mathbf{1 5}$ and $\mathbf{6}$ channels, does not suffer from the problem described here.

The final example we consider involves two kaons, for which the PQ extension has not been previously analyzed. For the $K K$ system, the PQ extension involves treating the two strange antiquarks as different, while the two light quarks can both be physical. Thus the graded symmetry group becomes $\mathrm{SU}(2) \times \mathrm{SU}(2 \mid 1)$, where the $\mathrm{SU}(2)$ is physical isospin. Thus PQ channels can be labeled by physical isospin, $I$, as well as "strange isospin," $I_{s}$, with both taking on the values 0 or 1 . For $s$-wave scattering, the overall symmetry implies that there are two allowed channels, with $\left(I, I_{s}\right)=(1,1)$ or $(0,0)$, which are, respectively, symmetric and antisymmetric under quark (or antiquark) exchange. Only the former is physical. Again, the sole unphysical channel, $(0,0)$, satisfies our criterion $\mathrm{C} 2$, since only contractions of type $D$ and $S$ are allowed as there is no annihilation in the $s$ channel. Thus the quantization condition could be applied to both channels.

\section{CONCLUSIONS}

This paper has demonstrated that the violation of unitarity in PQ theories at best restricts the application of the two-particle quantization condition to a subset of the unphysical two-particle channels. This has been demonstrated using $\mathrm{PQ} \chi \mathrm{PT}$, and our initial criteria $\mathrm{C} 1$ is stated within this effective field theory (EFT) framework. In this regard, we stress that the foundations of $\mathrm{PQ} \chi \mathrm{PT}$ as an effective theory for PQQCD are almost as strong as for QCD [19]. However, we have argued that $\mathrm{C} 1$ can be replaced by simpler, diagrammatic extension of the criterion, C2. This alternative version can be used irrespective of the existence of an EFT.

What is perhaps most surprising is that there may be unphysical two-particle channels in which the quantization condition can be used, because the derivation of the latter relies on unitarity, which is certainly violated in the PQ theory. The key observation here is that what matters for the derivation is that the corresponding scattering amplitude is unitary in the $s$ channel, while $t$ - and $u$-channel unitarity is 
not required. Thus for channels where the amplitude manifests its unphysicality only in the latter two channels, the problem that we raise does not occur.

We stress that, strictly speaking, we have not demonstrated this positive result, but rather only shown that one possible problem does not arise. However, we think it likely that one could extend the argument given here to all orders in the EFT, and that, for channels that satisfy our simple criteria, the use of the quantization condition is justified.

We also note that all is not lost in the channels that do not satisfy our criteria. One can still calculate the corresponding finite-volume correlators using $\mathrm{PQ} \chi \mathrm{PT}$, fit the results of a lattice simulation to the predicted form, and in this way determine the parameters of the EFT. This is the approach suggested in Ref. [5]. We stress, however, that our results show that one cannot bypass the problem by determining the energy shift solely from the term in the correlator ratio that is linear in $\tau$, and inserting the result into the twoparticle quantization condition, because the leading $c_{1} / L$ correction in that condition does not hold in PQ channels that do not satisfy our criteria.

Applying our criteria to the $\pi \pi$ and $K \pi$ systems, we find that the two previous applications which use results from simulations of LQCD are in unphysical channels that are not affected by the issue that we raise. Thus, the practical impact of our observation is to limit certain future applications of the approach of Refs. [13,14].

One way of understanding our central result is that, in a PQ theory, even if one starts with an operator that is composed of sea and valence quarks alone, in general there will be mixing with operators that include ghost quarks. In the calculation of Sec. III, this mixing was with operators of the form $\omega_{1 k} \omega_{k 2}$ and $\pi_{12} \phi_{1}$, both of which transform in the same 15 irrep of the SU(4) quark flavor group. One might hope that, by taking an appropriate linear combination of these operators, one could find a channel for which one could use the quantization condition. This amounts to classifying operators under the full $\mathrm{SU}(4 \mid 2)$ group, a task that we have not undertaken. We do not, however, expect this approach to be fruitful, since intermediate states will inevitably involve unphysical particles. For the same reason, we expect that the $\mathrm{SU}(4)$ singlet channel, where there can be mixing with several unphysical channels (including $\phi_{0}^{2}$ ), cannot be analyzed using the quantization condition.

A final comment concerns the foundational work of Bernard and Golterman [19]. They construct the transfer matrix of (latticized) PQQCD, showing that, while it is not Hermitian, it has a bounded spectrum. The import is that one expects correlation functions to still be given by a sum of exponentials as in Eq. (2), except the coefficients $c_{n}$ need not be positive. Furthermore, the energies $E_{n}$, while having real parts that are bounded from below, could be complex, although Ref. [19] argues that this is not the case for the lightest excitations in $\mathrm{PQ} \chi \mathrm{PT}$. Thus it could be that one can develop a generalization of the two-particle quantization condition that takes into account the possibility of negative $c_{n}$, and the concomitant cancellations. The lack of a simple exponential falloff that we have found could then be due to a cancellation between terms falling with similar exponents but opposite coefficients. The failure of the standard Lüscher threshold expansion could be due to the need to use an extension of the two-particle formalism. We do not know if such an approach will be fruitful, but it may be worth considering.

\section{ACKNOWLEDGMENTS}

We thank Maarten Golterman, Max Hansen, Ulf Meißner, and Fernando Romero-López for very helpful comments and suggestions. This work is supported in part by U.S. Department of Energy Award No. DESC0011637.
[1] C. W. Bernard and M. F. L. Golterman, Phys. Rev. D 49, 486 (1994).

[2] S. R. Sharpe, in Workshop on Perspectives in Lattice QCD (2006), arXiv:hep-lat/0607016.

[3] M. Golterman, in Les Houches Summer School: Session 93: Modern Perspectives in Lattice QCD: Quantum Field Theory and High Performance Computing (2009), arXiv: 0912.4042.

[4] S. R. Sharpe and N. Shoresh, Phys. Rev. D 62, 094503 (2000).

[5] M. T. Hansen and S. R. Sharpe, Phys. Rev. D 85, 054504 (2012).
[6] M. Della Morte and A. Juttner, J. High Energy Phys. 11 (2010) 154.

[7] N. R. Acharya, F.-K. Guo, U.-G. Meißner, and C.-Y. Seng, Nucl. Phys. B922, 480 (2017).

[8] J. C. Osborn, D. Toublan, and J. J. M. Verbaarschot, Nucl. Phys. B540, 317 (1999).

[9] L. Del Debbio, L. Giusti, M. Luscher, R. Petronzio, and N. Tantalo, J. High Energy Phys. 02 (2006) 011.

[10] S. R. Sharpe, Phys. Rev. D 74, 014512 (2006).

[11] P. H. Damgaard, J. C. Osborn, D. Toublan, and J. J. M. Verbaarschot, Nucl. Phys. B547, 305 (1999). 
[12] P. H. Damgaard, K. Splittorff, and J. J. M. Verbaarschot, Phys. Rev. Lett. 105, 162002 (2010).

[13] N. R. Acharya, F.-K. Guo, U.-G. Meißner, and C.-Y. Seng, J. High Energy Phys. 04 (2019) 165.

[14] C. K. Guruswamy, U.-G. Meißner, and C.-Y. Seng, Nucl. Phys. B957, 115091 (2020).

[15] S. R. Sharpe, R. Gupta, and G. W. Kilcup, Nucl. Phys. B383, 309 (1992).

[16] M. Lüscher, Commun. Math. Phys. 105, 153 (1986).
[17] M. Lüscher, Nucl. Phys. B354, 531 (1991).

[18] C. K. Guruswamy, U.-G. Meißner, and C.-Y. Seng, arXiv: 2102.03059.

[19] C. Bernard and M. Golterman, Phys. Rev. D 88, 014004 (2013).

[20] S. R. Sharpe and N. Shoresh, Phys. Rev. D 64, 114510 (2001).

[21] C. h. Kim, C. T. Sachrajda, and S. R. Sharpe, Nucl. Phys. B727, 218 (2005). 